\title{
Preparation of Graphene Nanopore based on AFM
}

\author{
Siyuan Wei, Zhongxin Qi, Tongyu Ji \\ Military Economics Academy of PLA, Wuhan City, Hubei Province, 430035, China
}

Keywords: Atomic force microscopy, EFM, LFM, TRM

\begin{abstract}
With the development of science and technology, graphene as a material has arrested increasing attention from scientists, and there are more experiments and researches on its application. The project is focused on the imaging technology of atomic force microscopy, which manages to conduct the image analysis of nanoscale materials such as graphene. It will also promote the understanding of various imaging techniques based on the atomic force imaging technology.
\end{abstract}

\section{Introduction}

In 1982, Dr. G.Binning, Dr. H. Rohrer and colleagues at IBM Zurich Research Laboratory invented STM (scanning tunneling microscope), which aims to conduct the research on the morphology and electronic structure of the surface with the use of tunnel current and can hence only be used to study samples of the semiconductor and subject matter but not to directly observe and study the samples of insulator and samples with thick oxide layers. In 1986 Binnig, Quate and Gerber invented the first AFM. With the continuous development of technology over the past few years, graphene related technology has been attached more importance to. It can be seen from the number of papers published in recent years that the interest in the research on graphene has been aroused. An important experimental instrument in the study of graphene, the atomic force microscopy boasts its own history of development and various types, and can be applied in different fields. The project aims to conduct research on the characteristics of AFM considering the conditions mentioned above.

\section{Concept of graphene}

1) Graphene is the planar honeycomb lattice of SP2 hybridized carbon atoms

2) Carbon atoms are connected through $\sigma$ bonds, which endow the graphene with highly excellent mechanical properties and structural rigidity

3) It is used for the manufacture of ultra-micro transistors, lubricants and additives, wear-resistant composite materials, new energy batteries, etc.

\section{Features of AFM}

1) The force between the tip of the probe on the micro-cantilever and the atoms of the measured samples is perceived and amplified, so as to achieve the purpose of detection; the atomic-scale resolution is a distinctive feature 
2) AFM provides a true three-dimensional surface map. For AFM, no special treatment of the sample is required. The atomic force microscope works properly under atmospheric pressure and even in the liquid environment

3) AFM has become one of the important tools for the study of biomedical samples and biological macromolecules

\section{Basic composition of AFM}

AFM perceives and handles the sample with the tip of the probe at the end of the cantilever of the microscope. AFM enables the quality of the sample surface or the pre-design of the nanostructures to be completed. AFM consists mainly of four parts, (i) AFM's probe. The role of the probe is to directly perceive and measure the force between the probe and the sample. AFM's probe is very sensitive to the interaction between atoms. Typically, there is a tip on the probe with length of less than $5 \mu \mathrm{m}$ and a diameter of less than $10 \mathrm{~nm}$. The probe is usually $100-500 \mu \mathrm{m}$ long; (ii) a piezoelectric scanner to precisely control the distance of the probe to the sample in the vertical and horizontal directions. The piezoelectric scanner is designed to bend, expand and connect a controlled and predictable operating platform when the voltage is appropriate; (iii) a professional software operating system to control the variables, so as to control the cantilever and probe, and to display and analyze the results; (iv) a feedback control system, including laser diodes, position photoelectric sensors and feedback circuits. The feedback system can detect and adjust the distance between the probe and the sample and serves as a communication tool between the piezoelectric scanner and the professional software.

\section{Basic operation form of AFM}

The probe is made from silicon nitride, and the cantilever is not movable, with which the resolution image of atoms can be obtained. The possible false appearance of the surface friction and viscosity may cause deformation of the surface and passivation of the tip.

The probe is made from silicon probe, featured by frequency modulation or phase modulation and with higher resolution; but because of the complex operation and interference of environmental noise, it is generally used in the test in a vacuum.

The probe made from silicon that can produce the imaging of soft samples. It can almost eliminate the reduction of resolution due to shearing force and the damage to the sample, and overcome the viscosity of the thin liquid layer on the surface of the sample at room temperature.

\section{AFM imaging}

High-resolution imaging can be used to analyze the morphology of specific nanomaterials, such as nanoparticles, nanotubes and nanosheets. The factors affecting the three-dimensional measurement and analysis of the nanomaterials are important. High-resolution imaging can also be used to analyze the surface roughness of certain nanomaterials, which is essential to compare the surface roughness of different nanomaterials.

Deformation imaging can be used to analyze the surface properties of nanomaterials when combined with the high-resolution imaging. The utilization is particularly effective in analyzing the surface properties of certain materials which are very flat, such as the surface of a cell. Recently, deformation imaging has been used to observe the protrusion of the nanoplate-like shape and the ripples on the surface of the nanosheet. 
Phase imaging is obtained in the intermittent contact or non-contact mode. The cantilever of AFM in the above two modes resonates with the piezoelectric actuator. During the imaging of AFM, the resonance of the cantilever changes with the distance from the sample to the tip. It is a mode of measuring the deformation of the cantilever during its periodic oscillations and the difference between the periodic signals as the piezoelectric actuator drives the cantilever. Phase imaging shows that the surface features of the sample can cause phase shift. Phase imaging has been widely used in researches on nanomaterials.

The lateral force imaging is obtained with the lateral force microscopy (LFM), also known as friction microscopy. LFM can achieve both high-resolution imaging and lateral force imaging, with similarity to the contact mode of AFM. In LFM, the scanning is carried out vertically along the long axis of the cantilever, and the force between the tip and the sample surface causes the torsion of the cantilever. With the lateral deviation, it acquires the information about the characteristics of the surface. LFM has now been widely used to describe the difference in frictions on the surface of nanomaterials. LFM imaging clearly shows the phase separation.

Magnetic gradient imaging is obtained with the magnetic force microscopy (MFM) and in the intermittent contact mode. By scanning the magnetic sample with a sharp magnetized tip, the magnetic interaction between the tip and the sample occurs when the tip contacts with the sample and reflects the magnetic gradient. MFM can detect the surface charge, surface potential, and interface potential distribution of the sample, the failure distribution of device, etc.

Torsional resonance imaging is obtained with the torsional resonance microscopy (TRM), which can measure and control the dynamic lateral force between the tip and the sample surface. InTRM, the cantilever vibrates around its long axis to form a torsional motion and further causes the vibration of the tip. When the tip sweeps through the surface of the sample, the vertical deflecting and lateral distortions of the cantilever are measured simultaneously. TRM boasts higher resolution, sensitivity and stability as compared with other modes of AFM.

CAFM is an effective current sensing method, which is obtained in the contact mode of AFM. In CAFM, when the tip sweeps over the surface of the sample, the DC bias is activated in the conductive tip and sample. CAFM is different from STM-STM is based on the concept of quantum tunneling. CAFM is useful in studying the surface properties of conductors and semiconductor nanomaterials.

Electric field gradient distribution imaging is obtained with the electrostatic force microscopy (EFM) and in the intermittent mode. In EFM, the voltage between the tip and the sample passes through when the tip contacts with the sample surface. In terms of typical EFM imaging, the phase, frequency, and amplitude of the oscillations of the cantilever are plotted in a planar Cartesian coordinate system. EFM can be used to analyze the electrical properties of the surface of nanomaterials.It can detect the surface charge, surface potential, and interface charge distribution of the sample, the failure distribution of device, etc.

Surface potential imaging is obtained with the scanning surface potential microscopy (SSPM) and in the intermittent mode.

\section{Achievements and conclusions}

With the search and reading of AFM related literature, I have a basic understanding of AFM and its derivatives as well as that of the mechanical properties of monolayer graphene at the nanometer level. In order to better and further carry out the study, I need to know more about the principle of some experiments, such as the method to test the cantilever deformation. I should also know more 
about the characteristics of the probe when in contact with the sample. Based on the above mentioned works, I am able to accomplish the operation.

\section{References}

[1] Jiang Zhong, Juan Yan. Seeing is believing atomic force microscopy imaging for nanomaterial research [J]. 2016

[2] Yang Yanlian, Introduction to atomic force microscopy [R]. 2007

[3] Nir Kampf. The use of AFM for soft matter studies[R]. 2011

[4] Ji Won Suk. Mechanical Properties of Monolayer Graphene Oxide [J]. 2010 